\title{
Situation of PMTCT in Bhutan, 14 Years' Experience
}

\author{
Phurb Dorji ${ }^{1}$, Ripa Chakma ${ }^{2}$, Lekey Khandu ${ }^{3}$ \\ ${ }^{1}$ Kidu Mobile Medical Unit, His Majesty's Secretariat, Thimphu, Bhutan \\ ${ }^{2}$ Faculty of Nursing \& Public Health, Khesar Gyalpo University of Medical Sciences of Bhutan, Thimphu, Bhutan \\ ${ }^{3}$ Department of Public Health, Ministry of Health, Thimphu, Bhutan
}

\begin{abstract}
Introduction: Prevention of mother-to-child transmission activities form important part of HIV/AIDS prevention program of any community or country. Methods: Information from the PMTCT Programme from the Ministry of Health and other published literature on HIV in Bhutan were reviewed. Results: The first case of mother to child transmission was reported in Bhutan in 2001. However, the retrospective study shows that one of the earliest mothers-to- child transmissions could have taken place back in 1997. Strategies for the prevention of mother-to-child transmission (PMTCT) of HIV in Bhutan have endured substantial advancement based on global scientific evidence. It is a concern as there is a slow rise in the number of HIV cases. The main mode of transmission is unsafe heterosexual practice in Bhutan. Before the planned PMTCT program, 3 children were infected. After launch of proper PMTCT program, we had all the components of effective strategies in PMCT program which have evolved with better ones with time. Due to this, MTCT was 3.2\%. With undetected HIV infections, 32 children were born outside of the program and actual national MTCT rate is 5.5\%. Conclusions: There is increasing number of people every year with new HIV infections. In addition, our detection gap is $45 \%$ with about 602 undiagnosed in the community. Every effort should be put forward to upscale the PMTCT program for Bhutan to eliminate Mother-to-Child transmission (MTCT) of HIV by 2020 and beyond.
\end{abstract}

Keywords: Bhutan; MTCT; PMTCT.

\section{INTRODUCTION}

Since the 1980s with over 33 years after detection, Human Immunodeficiency Virus (HIV) infection is one of the major causes of morbidity and mortality in the world. It is a global pandemic with over 37.9 million people estimated to be living with HIV across the globe ${ }^{1}$.

All countries and communities are affected. Each country has put up their strategies to fight the HIV infection supported by WHO since the early days. Bhutan has also responded early with establishment of the National HIV/AIDS and STIs Control Program (NACP) in $1988^{2}$. The first case of HIV infection in Bhutan was detected in $1993^{3}$. The National HIV/AIDS Commission was formed in 1993 to provide normative policy guidance in prevention and control of HIV/AIDS and STIs in the country ${ }^{2}$. Multi-Sectoral Task Force (MSTF) was set up in 2001 in all the 20 Dzongkhags, to ensure the participation of wider stakeholders to respond towards the prevention and control of HIV/AIDS which is still active to this day.

The Royal Decree on HIV and AIDS was issued by His Majesty the Fourth King on the $24^{\text {th }}$ of May 2004 for all members

\section{Corresponding author:}

Phurb Dorji

phurbd@gmail.com of the society to help prevent HIV and AIDS and provide care and compassion to those infected. In the same year the National HIV/ AIDS Commission endorsed giving of highly active antiretroviral therapy (HAART) for all the people living with HIV (PLHIV) from 2004 onwards.

Prevention of mother-to-child transmission (PMTCT) refers to interventions to avert vertical transmission of HIV from an HIV-positive mother to her baby during pregnancy, labor, delivery, or breastfeeding. Evidence shows that the combination of early diagnosis of infection, ART, safer obstetric practice and infant feeding counseling and support can reduce the risk of vertical transmission to less than $2 \%{ }^{4}$. The first case of PMTCT transmission case was detected in $2001^{5}$.

However, there are two periods when it comes to PMTCT status in Bhutan. There were no formal PMTCT program before 2005. The proper PMTCT was instituted from 2005 and it was integrated with the mother and child health clinic $(\mathrm{MCH})$ services in all the hospitals and is still going on well as of today. This article is an attempt to review the current PMTCT situation in Bhutan since the first case of HIV was diagnosed in 1993. It gives the account of PMTCT in two timeline periods

\section{PMTCT Before 2005}

There was no formal PMTCT program before 2005. Even HAART was very expensive and not available everywhere. All 
cases of HIV were monitored by CD4 counts and medications was provided through only one focal person for the whole country with top secrecy. There was only one single health worker who was given the task to look after all HIV infected cases and contact tracing. This was an effort to maintaining confidentiality to reduce stigma and discrimination of clients. There were times when the HIV infected people came to hospitals and received medical treatments and the treating physicians and nurses did not know their status. Even PMTCT cases only received single dose of nevirapine in labor and breastfeeding was routinely allowed. There were three children infected during this period whose mothers only received single nevirapine dose in labor and breast feeding was allowed.

\section{PMTCT after 2005}

In 2005, the Ministry of Health after receiving directives to start HAART for all the diagnosed PLHIV, health staff were selected and sent for training on clinical management of HIV and AIDS cases in Thailand. The teams comprised of Physicians, clinical nurses, and program managers who spent about two weeks each in Thailand supported by UNICEF and WHO.

The team members after returning from the training developed the Adult, Pediatric and PMTCT guidelines for the first time in Bhutan to put proper management plans into national action. In 2006 with the first PMTCT guideline, we managed to provide, routine opt-out voluntary counseling and testing (VCT) strategy to enhance case diagnosis, initiated HAART prophylaxis in pregnancy, and no breastfeeding policy by the HIV infected mother. There was strong disagreement from UNICEF to allow breast-feeding and finally UNICEF backed out financial support for printing of the first HIV and AIDS management guidelines including PMTCT when we kept our stand of no breast-feeding in our strategy. However, Ministry of Health got the guidelines published and multiple trainings were conducted for field staff covering all Dzongkhags.

PMTCT program has been started as an integrated component of the reproductive health of the mother and child. HIV test was routinely offered in the antenatal clinics to all women coming for ANC through opt-out approach. Our initial three PMTCT specific strategies were giving free prophylactic ART to the HIV infected pregnant mothers and to newborns, adoption of safe vaginal delivery practices were promoted to reduce contact of newborn with maternal body fluids in labor and delivery and maintained no breastfeeding from the beginning. All HIV infected mothers were supported with distribution of free infant formula for baby feeding initially for one year and now for 2 years by Ministry of Health. However, breast feeding in general population was actively promoted as per the national exclusive breast-feeding policy. From 2019 we have 6 months of maternity leave for all mothers to support exclusive breast feeding for general population. Based on the many recommendations made by WHO, PMTCT approach in Bhutan has also changed accordingly as shown in Figure 1, below. Until 2010, we have allowed for vaginal delivery of all HIV infected mothers and caesarean section was done only for obstetric indications. From 2010 with starting of triple ART prophylaxis from 14 weeks, the PMTCT program in Ministry of Health has started to offer elective caesarean section at 39 weeks and continues till date. Infants are given ART prophylaxis after birth since 2006.

\section{Situation of PMTCT till 2019}

Out of these in total we have 111 mothers identified as PMTCT cases till 2019 Since 2004, we have accurate data for annual PMTCT cases detected. Figure 2 shows the annual PMTCT cases detected since 2004 until 2019 in Bhutan.

\section{Age of mothers}

From the total 687 HIV infection detected cases in the country, the majority $(58 \%, n=14)$ of them are between the ages of 25-49 while $4 \%(n=1)$ are between $15-24$ years of age and $38 \%(n=9)$ above 50 years 6 .

The mean age of the mothers (PMTCT cases) is 26.9 years ( \pm 5.3 years) and it is similar to the general group. Figure 3 below shows the age distribution of PMTCT cases. It is affecting the age group where there is active reproductive life and income earning age. The double burden is that there is risk of more spread in the community with young age of HIV infected females and if they don't or fail to use condom regularly. The other burden is that young working age group being affected hinders income generation for the family and community.

\section{Occupation}

As shown in Figure 4 below, it is very saddening to see that the majority $(70.4 \%)$ of the PMTCT cases belong to housewives followed by farmers and others. It may reflect that HIV infection in Bhutan has reached maturation level where infection has

\begin{tabular}{llll}
\hline Year & $\mathbf{2 0 0 6 - \mathbf { 2 0 1 0 }}$ & $\mathbf{2 0 1 0 - \mathbf { 2 0 1 3 }}$ & $\mathbf{2 0 1 3 - \mathbf { 2 0 1 8 }}$ \\
\hline ARV & ZDV single dose & ZDV/3TC/NVP & TDF+3TC+EFV \\
Period of Gestation & 28 weeks & From 14 weeks & As Early as possible \\
Mode of Delivery & Vaginal delivery & Elective Caesarean section & Elective Caesarean section \\
Infant Feeding & No Breast Feeding & No breastfeeding & No breastfeeding \\
\hline
\end{tabular}

Figure 1. To show the PMTCT Interventions 


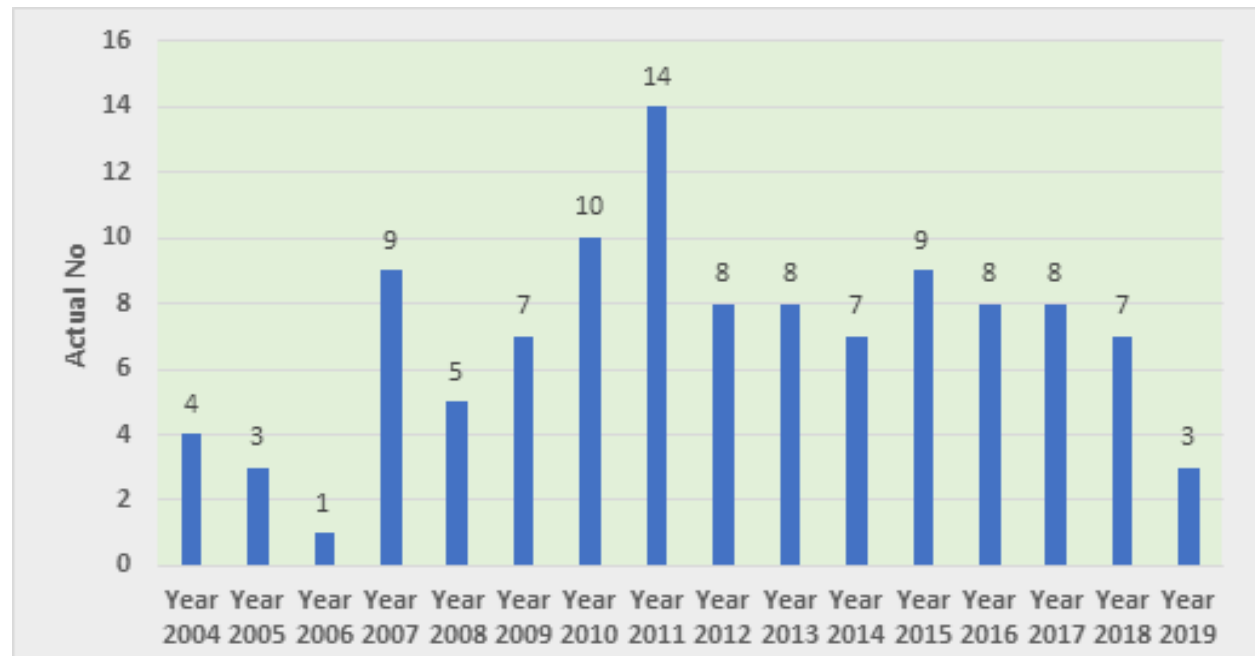

Figure 2. The annul HIV cases among the pregnant Bhutanese mothers from 2004-2018

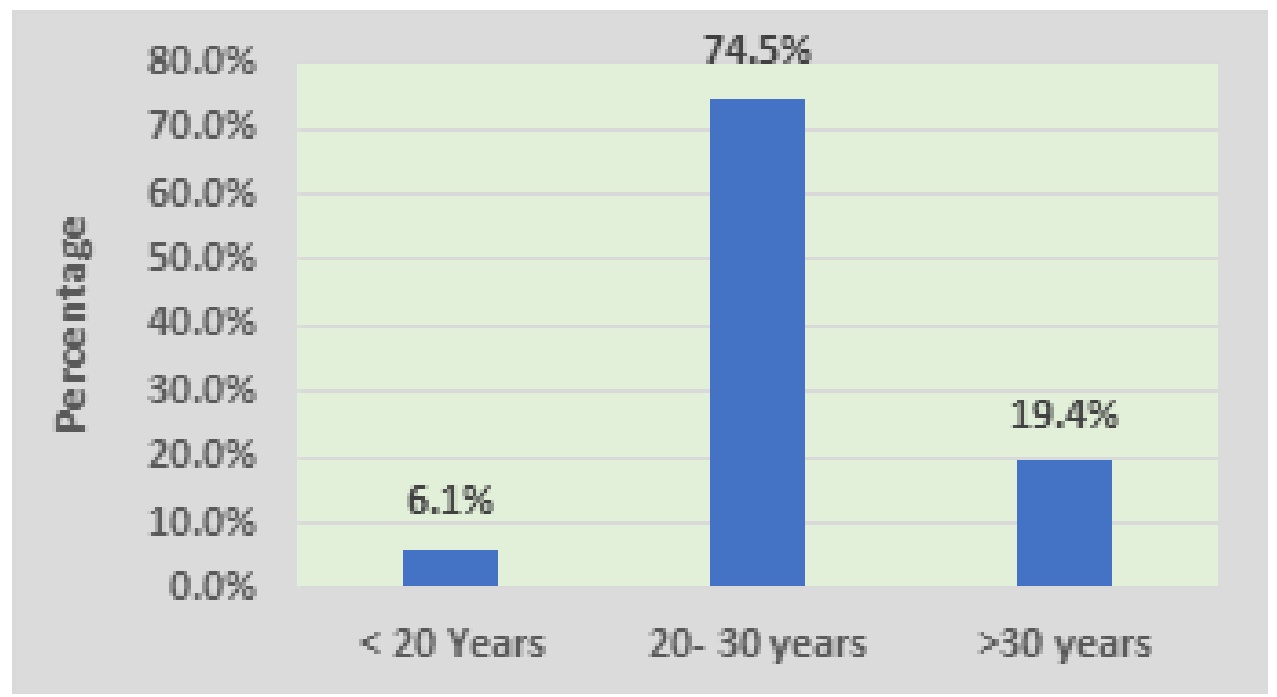

Figure 3. Age distribution of HIV positive pregnant women in Bhutan from 2004 to 2018

reached even to the housewives and farmers. It may also be that it is the problem of routine surveillance not being able to capture the appropriate risk behavior at the time of diagnosis due to no case-based surveillance instituted that point in time.

\section{Mode and place of delivery}

The majority of the PMTCT mothers had vaginal delivery because the caesarean delivery has started only from 2010 based on the WHO recommendation to gear toward zero vertical transmission. This is shown in Figure 5. As a result, there has been significant drop in number of women delivering vaginally after 2010 .

Majority (51.2\%) of the total cases of PMTCT patients have delivered in JDWNRH. This was due to the prevalence of good practice from the very beginning from 2006 regarding care of PMTCT cases including maintaining full privacy and confidentiality in JDWNRH. Secondly, many mothers had relatives living in Thimphu who provided support during hospitalization and delivery but after the introduction of the policy of caesarean section delivery, many have delivered in hospitals with gynecological services in other districts.

\section{HIV Status of Children through the PMTCT}

It remains a challenge for the PMTCT program at present that early infant diagnosis facilities are not available in the country. We need to wait till 18 months after delivery to confirm the status of the babies. This is a long period of waiting and many things are likely to happen.

Before the start of the proper PMTCT program, before 2006, there were 7 PMTCT cases who only received single dose of NVP in labor and had breast feeding. From here 3 children were positive and MTCT was $42.8 \%$. This picture is as good as having not any intervention in place.

From start of proper PMTCT program from 2005 to 2019, 98 children were born. 1 child died before completion of 


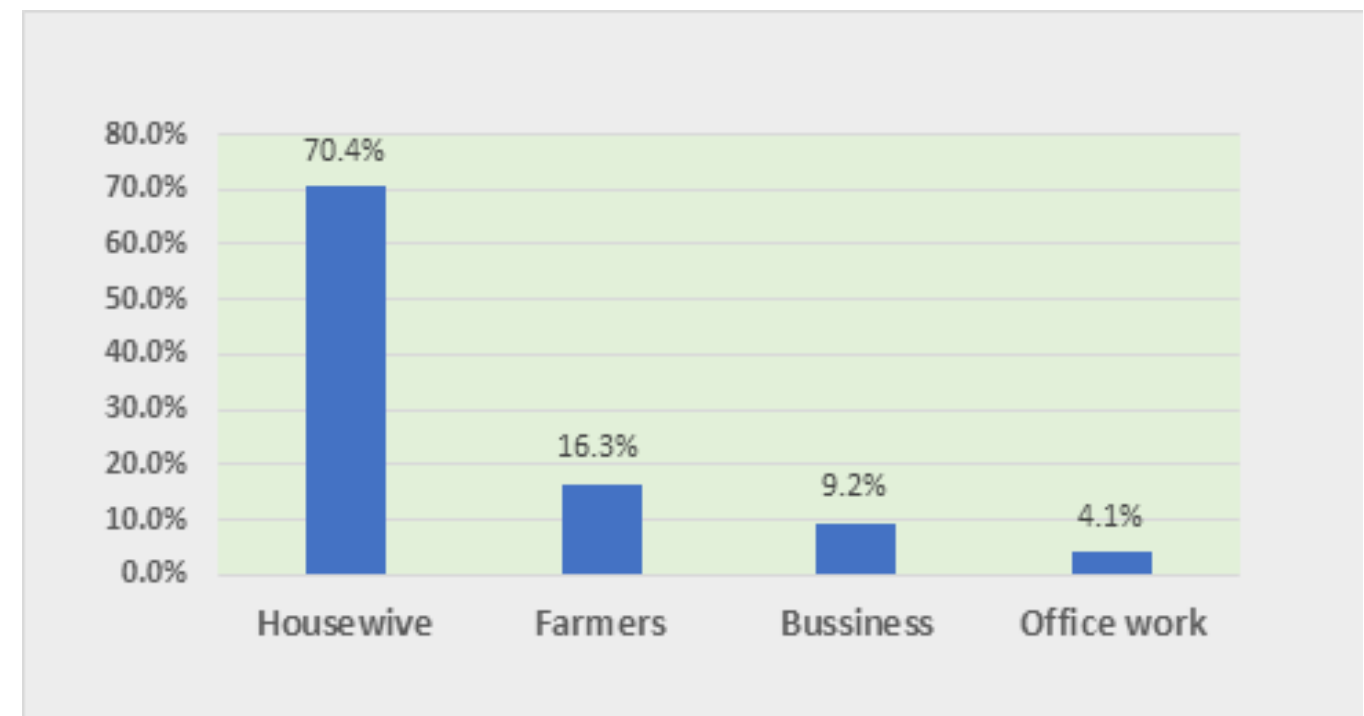

Figure 4. Occupation of HIV positive pregnant women in Bhutan from 2004 to 2018

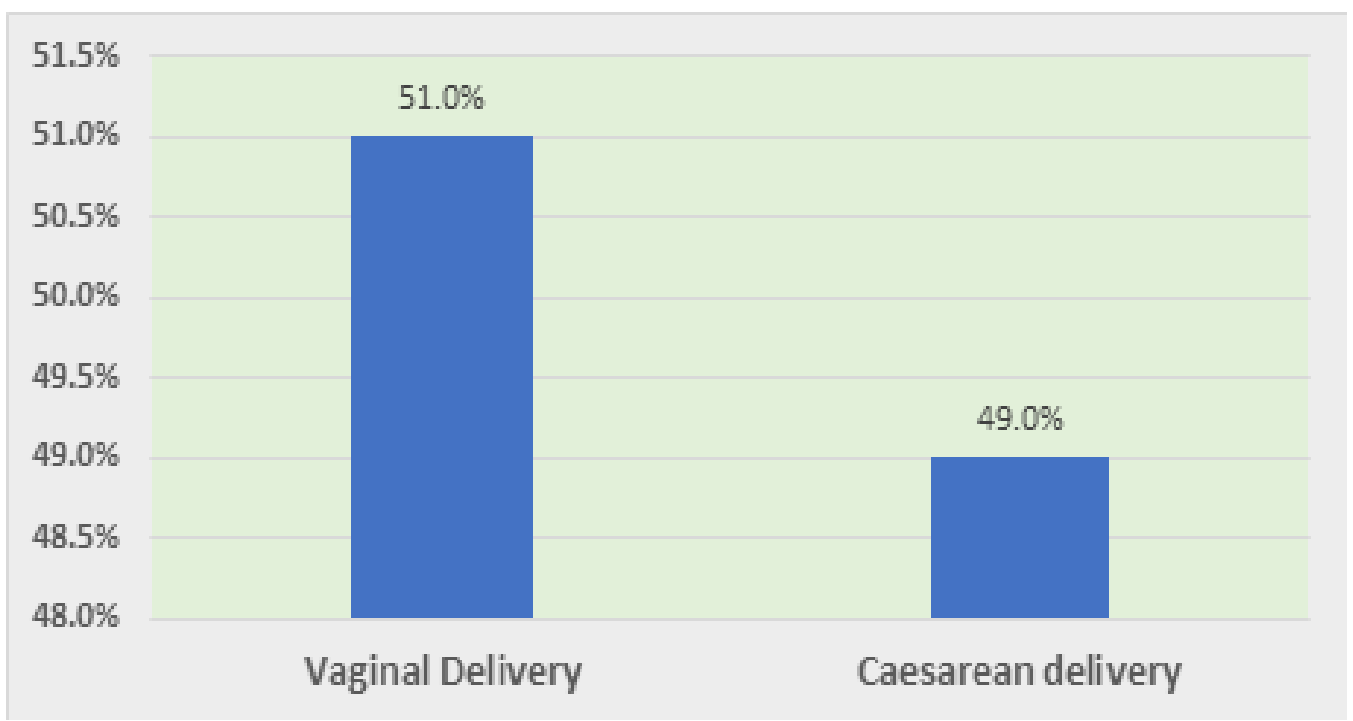

Figure 5. Mode of delivery of HIV positive pregnant women in Bhutan from 2004-2019

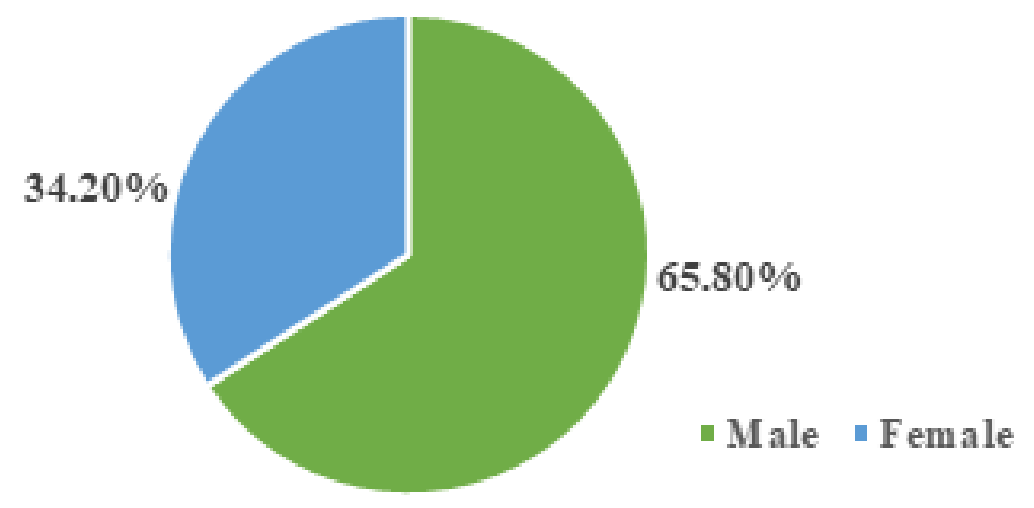

Figure 6. To show sex of the children 


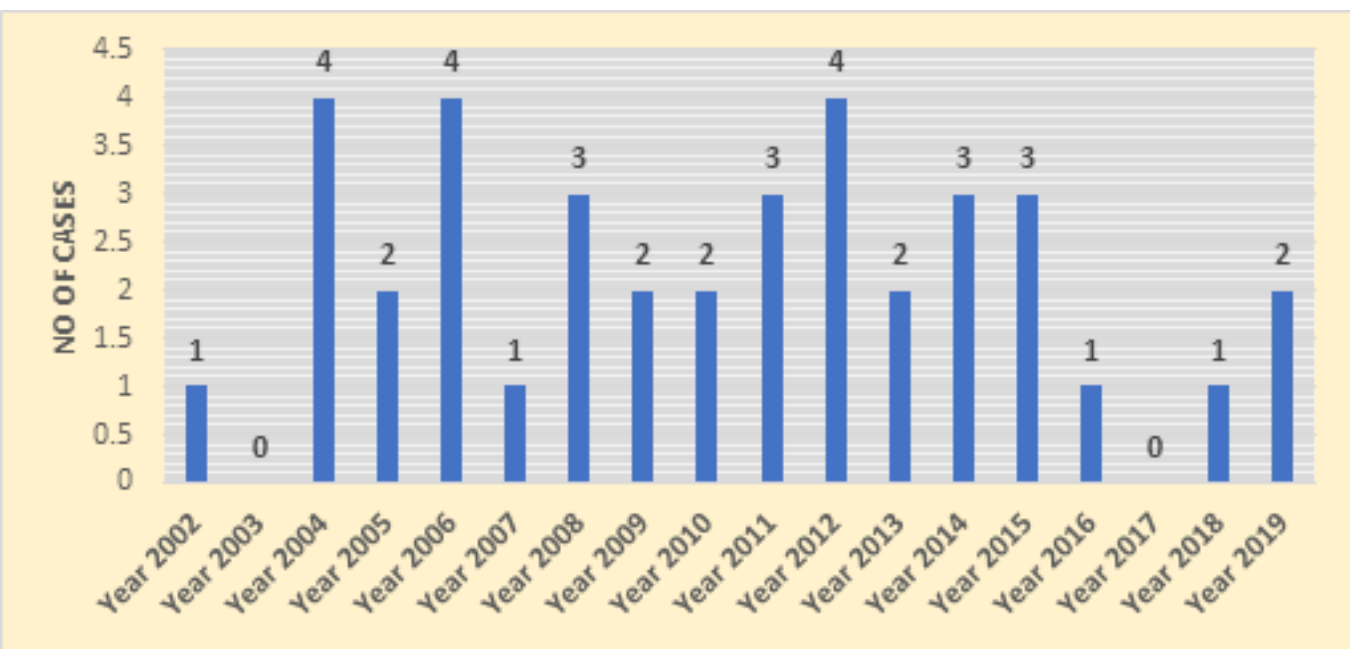

Figure 7. Annual PMTCT transmission

follow up and 2 were lost to follow up. 95 out of 98 children were tested negative and the MTCT rate was 3.2\% through the proper PMTCT program where death before testing and lost to followup were taken as positive cases.

From 2001 to 2019, in addition to 6 cases detected through the PMTC program from 2001 to 2019, there were 32 children born from positive mothers who were not registered in the PMTCT program as they did not know their status. All of these were diagnosed through contact tracing of newly diagnosed cases of HIV cases. This puts the total PMTCT cases as 111 and MTCT as 38 cases. Out of 38 cases 2 children have left outside the country as it being non-national and 4 children have died. Thus, the prevalence of MTCT in Bhutan at present is 5.5\%. Currently there are 32 children living with HIV in Bhutan.

Majority of children of MTCT cases were male (65.80\%) as shown in Figure 6.

The annual number of MTCT cases detected both in the program and outside the program is shown in Figure 7.

\section{DISCUSSION}

The first case of mother to child transmission was reported in Bhutan in $2001^{5}$. However, the retrospective study shows that one of the earliest mothers - to- child transmissions could have taken place back in 1997.

The total cumulative HIV positive cases reported since 1993 until November 2019 stands at 687 (359 male and 328 female). Out of this, 38 HIV positive cases are from MTCT. Thus the prevalence of MTCT in Bhutan at present is $5.5 \%$.

Since the start of proper PMTCT program the record of $3.2 \%$ MTCT cases is an indication of good PMTCT case management in Bhutan. This is evident as there is no MTCT cases for last two to three years expect few cases that have not received any PMTCT services during their entire pregnancy and at the time of delivery. This shows that Bhutan is in verge of elimination within a few years from now if we could get all the women diagnosed in pregnancy. This global finding also shows that initially testing HIV negative and subsequently testing positive in pregnancy or breastfeeding (leading to delays in ART initiation) is a major cause of PMTCT failure, occurring in $8(42.1 \%)$ of the PMTCT failure cases ${ }^{7}$. One effective intervention put in place to respond appropriately to prevent MTCT is case management with the provision of free lifelong triple drug treatment for all mothers including the mothers who have delivered before. However, we have a HIV detection gap of $47 \%$ of the estimated 1265 HIV cases in Bhutan ${ }^{6}$. The existence of this group has shown that 32 children were born to the undetected group pushing the national level of MTCT to 5.5\%. Furthermore, the current case detection gap may also contribute to more HIV positive pregnant mothers in coming years which is evident from the current reported cases highest cases belongs to housewives ${ }^{6}$. Therefore, if the current PMTCT interventions are not scaled up with 100\% institutional delivery and strong mechanism of following the HIV negative pregnant mother with repeat HIV test in third trimester of pregnancy and after delivery to prevent post exposure, then the likelihood of jeopardizing the current gains in triple elimination of MTCT of HIV, Congenital Syphilis and Hepatitis B is high. This is evident from the findings where late seroconversion in pregnancy or postpartum, and some health system-related factors played a critical role in failure of the PMTCT programs in many countries in the region?

The finding from the global literature also depicts that mother's place of residence, infant's place of delivery, infant ARV prophylaxis at birth, feeding practice, infant age at enrollment, maternal enrollment into care, and PMTCT mother-child pairs were all associated with $\mathrm{MTCT}^{8}$. Within the range of these determinants of Maternal to MTCT of HIV the mothers receiving HIV education including counseling and engaging their spouse in disclosure of the status is one element to be considered. In addition, it was also shown that mothers who lacked any social support were also 2.83 times more likely to bear an HIV-infected infant ${ }^{8}$. The current planned allowance for all pregnant mother 
in Bhutan by the Royal Government of Bhutan if implemented as planned would enable Bhutan to achieve above $95 \%$ institutional delivery thus minimizing the MTCT cases.

The other aspect of PMTCT in Bhutan is that until 2018, there is no confirmatory test if the first test is negative. This leaves many with possible acute phase or late seroconversion cases in some women. This is supported by one the study done in Durban found that $2.1 \%$ of patients with a negative rapid HIV test had either acute HIV infection (which was missed by the rapid test because of falling within the 'window period') or chronic HIV infection (i.e. a false negative rapid test) 9 . Late booking after 20 weeks' gestation (also leading to late initiation of maternal ART) is one cause of failure in PMTCT $^{8}$. Virus develops resistance to the medications in few cases. Infants born through home delivery were 2.40 times more likely to be HIV infected ${ }^{8}$.

In Bhutan, about $16 \%$ with MTCT had home delivery and this has put the PMTCT program at greater risk where infection spread remains a huge threat. Home delivery against medical advice could have been one of the deliberate attempts of the mothers or couple to maintain privacy for fear of social stigma and discrimination.

\section{The way forward}

PMTCT is a very good integrated program with $\mathrm{MCH}$ in Bhutan. Better understanding of the reasons for MTCT can assist further reduction of MTCT rates to the target of less than $2 \%$.

To keep our situation not only under control but efforts are moving towards action for Bhutan to eliminate Mother-toChild transmission (MTCT) of HIV by 2020 and beyond ${ }^{6}$. This is in line for Bhutan to gear towards the triple elimination of HIV, Syphilis and hepatitis B among the newborn by 2020 and beyond ${ }^{10}$.

Our institutional delivery has reached $96 \%$. Some of the new initiatives towards prevention of HIV and AIDS in the country are introduction of mobile HIV testing and counselling services, installing of condom vending machines in the priority districts to promote condom use. Rolling out of treat all policy to ensure all those diagnosed with HIV on ART treatment and introducing the viral load services to monitor the treatment efficacy.

Strategies to increase detection of all positive cases could include a second confirmatory rapid test as routine in all pregnant women (with an HIV ELISA confirmatory test for all discordant results). This could be made as regular 3-monthly maternal HIV testing throughout the duration of pregnancy. This will be the best option to include all pregnant women.

The other key intervention is the strong mechnisim to follow those negative pregnant mother after their deliver to prevent the post delivery exposure.

The need to initiate the early infant diagnosis facility is very important to enable the the country gear towards elimination as it is one of the perquisite as per the WHO guidelines for elimination.
Further research is needed to characterise the frequency of false-negative HIV testing in operational PMTCT programmes.

\section{CONCLUSIONS}

PMTCT program is very important to reduce transmission of HIV in the community. We need to tighten and improve our health service delivery system to improve further. Failure of this program adds another generation of people with HIV positive in the community, increasing the risk much higher. The Program needs to adopt multiprong approach to have all pregnant mothers tested more than once in antenatal period. Detection is the key to prevention.

\section{REFERENCES}

1. Factsheet - Global aids update 2019. [Full Text]

2. National STI \& HIV/AIDS Control Program, Ministry of Health, Bhutan. National strategic plan for the prevention and control of STIs and HIV and AIDS. 2008. [Full Text]

3. National STI \& HIV/AIDS Control Program, Ministry of Health, Bhutan. PLHIV database; 2016. Data provided by the record manager in excel sheet.

4. Cock KMD, Fowler MG, Mercier E, Vincenzi Ide, Saba J, Hoff E, et al. Prevention of mother-to-child HIV transmission in resource-poor countries: translating research into policy and practice. JAMA. 2000 Mar 1;283(9):1175-82. [Full Text | DOI]

5. UNICEF Bhutan. Epidemiological fact sheets. HIV/AIDS and sexually transmitted infections. Bhutan. UNICEF Bhutan. 2006. [Full Text]

6. Press Release, National STI \& HIV/AIDS Control Program, Ministry of Health, Bhutan, Nov 2019.

7. Kendall C, Claessens L, Dorward J, Mfeka G, Gate K. Reasons for failure of prevention of mother-to- child HIV transmission in a rural South African district hospital. S Afr J HIV Med. 2015;16(1). [Full Text | DOI]

8. Wudineh F, Damtew B. Mother-to-Child Transmission of HIV Infection and its determinants among exposed infants on care and follow-up in Dire Dawa City, Eastern Ethiopia. AIDS Research and Treatment. 2016. [Full Text | DOI]

9. Johnson LF, Stinson K, Newell ML, Bland RM, Moultrie $\mathrm{H}$, Davies MA, et al. The contribution of maternal HIV seroconversion during late pregnancy and breastfeeding to mother-to-child transmission of HIV. J Acquir Immune Defic Syndr. 2012 April 1; 59(4): 417-425. [PubMed | Full Text | DOI]

10. National STI \& HIV/AIDS Control Program, Ministry of Health, Bhutan. National HIV, AIDS and STI Strategic Plan 2017-2023. [Full Text] 